\title{
VALIDAÇÃO DE UMA NOVA FUNÇÃO DE AUTOCORRELAÇÃO PARA DESCREVER O MEANDRO DO VENTO HORIZONTAL
}

\author{
Lilian Moor ${ }^{1}$, Gervasio Annes Degrazia ${ }^{1, *}$, Lidiane Buligon ${ }^{2}$, Charles Szinvelski ${ }^{2}$, Otávio Acevedo ${ }^{1}$, \\ Silvana Maldaner ${ }^{1}$, Marco Túllio Menna Barreto De Vilhena ${ }^{3}$ \\ ${ }^{1}$ Universidade Federal de Santa Maria, Departamento de Física, Santa Maria-RS, Brasil. \\ ${ }^{2}$ Universidade Federal de Santa Maria, Departamento de Matemática, Santa Maria-RS, Brasil. \\ ${ }^{3}$ Universidade Federal do Rio Grande do Sul, Instituto de Matemática, Porto Alegre-RS, Brasil. \\ *gervasiodegrazia@gmail.com
}

Summary: The aim of this work is to employ observational data to evaluate a new autocorrelation function describing meandering phenomena.

Resumo: O objetivo deste trabalho é validar experimentalmente uma nova função de autocorrelação para o fenômeno de meandro do vento.

\section{INTRODUÇÃO}

O Meandro do vento é um fenômeno associado com a turbulência na camada limite planetária. Este fenômeno ocorre quando a velocidade do vento está abaixo de $1.5 \mathrm{~m} / \mathrm{s}$ (Anfossi, 2005). Durante a condição de meandro, a direção horizontal do vento varia muito e os contaminantes são dispersos sobre muitos setores angulares. Consequentemente, o comportamento oscilatório do vento é o mecanismo que espalha os contaminantes nos diferentes ângulos e provoca uma redução na concentração máxima destes (Degrazia et al., 2008). Frenkiel (1953) propôs uma função de autocorrelação para descrever o meandro e percebeu que estas funções de autocorrelação para as componentes laterais do vento apresentam um comportamento oscilatório com a presença de grandes lóbulos negativos (Anfossi et al. 2005, Oettll et al. 2005).

O objetivo do presente estudo é validar a função de autocorrelação sugerida por Degrazia1 utilizando dados experimentais coletados na campanha LBA. Adicionalmente, a mesma expressão será comparada com a função de autocorrelação proposta por Frenkiel.

11 Comunicação pessoal Degrazia, G. A., Santa Maria, setembro/2013. 


\section{FUNÇÕES DE AUTOCORRELAÇÃO}

Frenkiel (1953) propôs a seguinte forma para a função de autocorrelação,

$$
R_{1}(\tau)=e^{-p \tau} \cos q \tau
$$

com $\mathrm{p}=\frac{1}{\left(\mathrm{~m}^{2}+1\right) \mathrm{T}_{\mathrm{Lu}_{\mathrm{u}}}}$ e $\mathrm{q}=\frac{\mathrm{m}}{\left(\mathrm{m}^{2}+1\right) \mathrm{T}_{\mathrm{L}_{\mathrm{u}, \mathrm{v}}}}$ (Murgatroyd, 1969). onde $p$ e $q$ são parâmetros híbridos expressos em termos de $\mathrm{T}_{\mathrm{L}_{\mathrm{u}, \mathrm{v}}}$ (escala de tempo para uma turbulência bem desenvolvida) e $m$ uma quantidade adimensional que controla a frequência de oscilação do meandro do vento horizontal (Anfossi et al., 2005). Em seus estudos Degrazia propôs uma nova função de autocorrelação, dada pela equação:

$$
R_{2}(\tau)=\frac{\cos (q \tau)}{(1+p \tau)^{2}}
$$

A Eq. (2) está em processo de estudo teórico e neste trabalho será comparada com um primeiro conjunto de dados experimentais.

\section{DADOS E RESULTADOS}

Os dados utilizados para o estudo foram coletados em Santarém na Amazônia brasileira através do Experimento de Grande Escala da Biosfera-Atmosfera na Amazônia (LBA) (Alencar, et. al., 2010). As Figuras 1 e 2 mostram os resultados da comparação entre os dados experimentais do meandro do vento (linha em cinza) e as funções de autocorrelação de Frenkiel (Eq. (1) - linha tracejada) e Degrazia (Eq. (2) - linha contínua), respectivamente. Os gráficos (Figuras 1 e 2) referem-se aos dados de período noturno com velocidades horizontais do vento menores que $1,5 \mathrm{~m} / \mathrm{s}$.

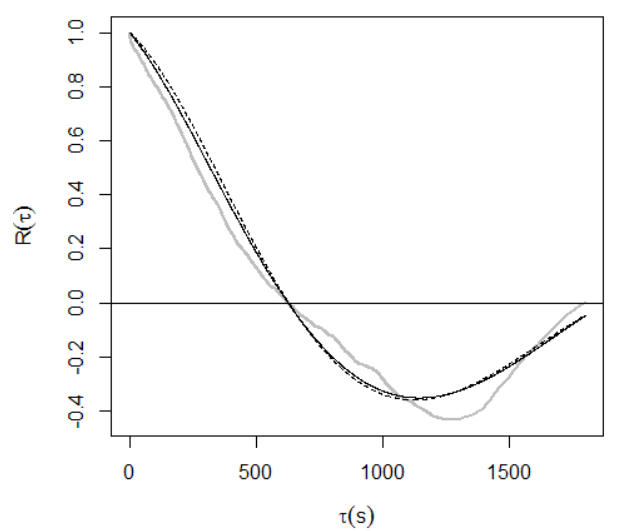

Figura 1 - Comparação entre as funções de autocorrelação para o dia 21 de abril de 2001, $23 \mathrm{~h}$. A linha cinza refere-se aos dados de Santarém (componente v do vento), a linha tracejada representa a função de autocorrelação de Frenkiel (Eq. (1)) e a linha contínua representa a função de autocorrelação de Degrazia (Eq. (2)). 


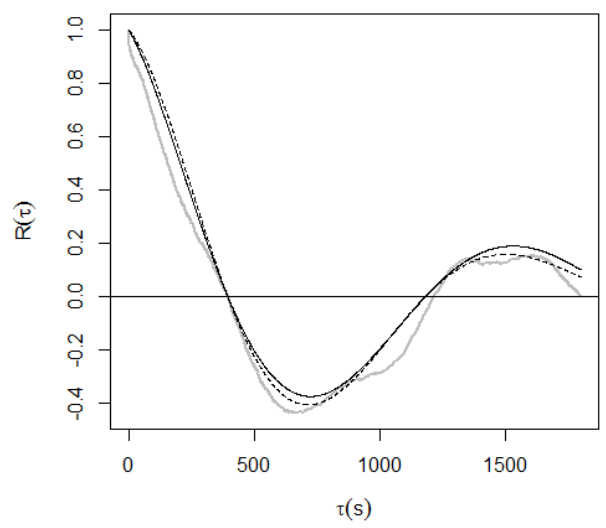

Figura 2 - Comparação entre as funções de autocorrelação para o dia 21 de abril de 2001, $21 \mathrm{~h}$. A linha cinza refere-se aos dados de Santarém (componente u do vento), a linha tracejada representa a função de autocorrelação de Frenkiel (Eq. (1)) e a linha contínua representa a função de autocorrelação de Degrazia (Eq. (2)).

\section{CONCLUSÕES}

Os resultados sugerem que a nova função de autocorrelação (Eq. 2) representa, satisfatoriamente, os dados experimentais e reproduz a expressão proposta por Frenkiel.

\section{AGRADECIMENTOS}

A CAPES, CNPQ e FAPERGS.

\section{REFERÊNCIAS}

ALENCAR, A. L. AND CAMPOS DOS SANTOS J. L., Um Ambiente para Tratamento Automático de Dados Micrometeorológicos do Projeto LBA. In: II Escola Regional de Informática da Região Norte I, Manaus, 2010.

ANFOSSI, D., OETTL, D., DEGRAZIA, G. A., AND GOULART, A., An analysis of sonic anemomenter observations in low wind speed conditions, Bound.-Lay. Meteorol., v.114, pp.179-203, 2005.

CSANADY, G. T., Turbulent Diffusion in the Environment, Geophysics and Astrophysics Monograps, Reidel, Boston, 248 p., 1973.

DEGRAZIA, G. A.,GOULART, A.,COSTA CARVALHO, J., $\quad$ SZINVELSKI, C. R. P., BULIGON, L., UCKER TIMM, A., Turbulence dissipation rate derivation for meandering occurrences in a stable planetary boundary layer. Atmospheric Chemistry and Physics, v.8, pp.1713-1721, 2008. 
FRENKIEL, F. N., Turbulent diffusion: Mean concentration distribution in a flow field of homogeneous turbulence, Adv. Appl. Mech., v.3, pp.61-107, 1953.

MURGATROYD, R. J., Estimations from Geostrophic Trajectories of Horizontal Diffusivity in the Mid-Latitude Troposphere and Lower Stratosphere, Quart. J. Roy. Meteorol. Soc., v.95, pp.40-62, 1969.

OETTL, D., GOUlART, A., DEGRAZIA, G., AND ANFOSSI, D., A new hypothesis on meandering atmospheric flows in low wind speed condition, Atmos. Environ., v.39, pp.1739-1748, 2005. 